\title{
Integrierte Gesundheitsversorgung: eine unwahrscheinliche Leistung
}

\author{
Die integrierte Gesundheitsversorgung gilt als eine Möglichkeit, den aktuellen \\ Herausforderungen des Gesundheitswesens zu begegnen. In diesem zweiteiligen \\ Beitrag beleuchten wir, warum sie eine höchst unwahrscheinliche Errungenschaft \\ darstellt. Und was dennoch getan werden könnte, um die Wahrscheinlichkeit ihres \\ Gelingens zu erhöhen. Der zweite Teil erscheint in der nächsten SÄZ-Ausgabe.
}

\section{Matthias Mitterlechner ${ }^{a}$ Johannes Rüegg-Stürm ${ }^{b}$, Harald Tuckermann ${ }^{c}$}

a Dr. oec. HSG, Habilitand und Co-Leiter Forschungsprogramm HealthCare Excellence, Institut für Systemisches Management und Public Governance an der Universität St. Gallen (IMP-HSG)

b Prof. Dr. oec., Direktor des Instituts für Systemisches Management und Public Governance an der Universität St. Gallen

c Dr. oec. HSG, Habilitand und Co-Leiter Forschungsprogramm HealthCare Excellence, Institut für Systemisches Management und Public Governance an der Universität St. Gallen (IMP-HSG)

* Die Literaturangaben finden sich unter www.saez.ch $\rightarrow$ Aktuelle Ausgabe $\rightarrow$ Inhal oder $\rightarrow$ Archiv $\rightarrow 2013 \rightarrow 26$

Korrespondenz:

Prof. Dr. oec.

Johannes Rüegg-Stürm Institut für Systemisches

Management und

Public Governance

Dufourstrasse 40a

CH-9000 St. Gallen

Tel. 0712242323

Fax 0712242536

johannes.rueegg[at]unisg.ch

www.hce.imp.unisg.ch

www.healthcaremanagement.ch

\section{Die Unwahrscheinlichkeit integrierter Versorgungsnetze}

Die integrierte Gesundheitsversorgung ist in aller Munde. Auf Symposien und Fachtagungen sowie in Artikeln und Expertenberichten wird fortlaufend darauf hingewiesen, wie dringlich innovative Versorgungsmodelle sind, um akuten Herausforderungen im Gesundheitswesen wie der demographischen Alterung, der starken Zunahme chronischer, multimorbider Erkrankungen und der wachsenden Schere zwischen dem medizinisch Machbaren und finanziell Verkraftbarem wirksam zu begegnen. Im Kontext dieser Veränderungen hängt eine erfolgreiche Patientenbehandlung immer weniger von der kompetenten Intervention eines einzelnen Spezialisten ab, sondern zunehmend von Versorgungsstrukturen, in denen mehrere Professionen organisationsübergreifend auf partnerschaftlicher Basis zusammenarbeiten [1]*. Die Verlagerung der Wertschöpfung ins Netzwerk dient dazu, Expertise zu bündeln, Schnittstellen zu optimieren, Doppelarbeiten zu reduzieren und die Patienten wirksamer und wirtschaftlicher $\mathrm{zu}$ behandeln (siehe dazu auch [2]). Zwischen diesem diskursivem Konsens und der Umsetzungsrealität klafft jedoch eine beträchtliche Lücke: «Zumindest in den Meinungsäusserungen scheint ein breiter Konsens zu bestehen, dass die Inhalte, Rahmenbedingungen und Zuständigkeiten der Berufe im Gesundheitswesen sich wandeln und entwickeln müssen, doch viel weiter hat dieser etwas ratlos anmutende Konsens noch nicht geführt» [3]. Neue Gesundheitszentren, Ärztenetze und regionale Projekte werden zwar regelmässig als vielversprechende Zukunftsmodelle präsentiert, allerdings «... wird eine verbesserte Integration und Vernetzung sowie Koordination in den Modellen nur zum Teil anvisiert oder umgesetzt» [2].

Wir führen diese Situation nicht auf den fehlenden Mut der Beteiligten zurück, sondern ganz im Gegenteil auf die enorme Komplexität, die bei einer inter-organisationalen Zusammenarbeit zwischen den Professionen des Gesundheitswesens zu bewältigen ist. Beispielsweise ist Kooperation nicht nur mit Erträgen, sondern stets auch mit Aufwänden wie

\section{La couverture de soins intégrés: une performance peu vraisemblable}

En permanence, des experts soulignent combien il est important de disposer de modèles de soins innovants pour faire face aux défis actuels du système de santé. Mais un abîme sépare le consensus discursif et la mise en œuvre concrète. Ce n'est pas au manque de courage des personnes concernées que nous imputons cet abîme, mais à l'énorme complexité d'une collaboration inter-organisationnelle. D'une part, il est peu probable que ce sujet soit au centre de l'attention des fournisseurs de prestations. En raison de la fragilité de leur création de valeur, leur attention est dirigée principalement vers la stabilisation de l'interaction immédiate médecin-soinspatient, et moins vers les questions de conception inter-organisationnelle du processus de création de valeur. Même si les fournisseurs de prestations parviennent à prêter davantage d'attention à une coopération inter-organisationnelle, il reste peu probable que les professions représentées au sein du réseau puissent se comprendre. Au cours de leur différenciation les unes des autres, elles ont en effet développé des pratiques très spécifiques qui ne se laissent réintégrer qu'au prix d'importants efforts de communication. Enfin, il est peu probable que professions et patients s'engagent fermement en faveur d'une coopération dans le réseau, tout réseau limitant les libertés de choix et produisant une complexité factuelle et sociale difficile à gérer pour les personnes concernées. 
einem teilweisen Verlust von Handlungsautonomie verbunden. Zudem bedingt Kooperation einen reibungslosen Informationsfluss zwischen den Beteiligten und schafft dadurch Transparenz über alle Netzwerkpartner, welche vor allem in Wettbewerbsverhältnissen ambivalent sein kann [4]. In diesem Beitrag machen wir uns auf eine Spurensuche und versuchen, tiefer zu ergründen, warum integrierte Versorgungsmodelle in der Praxis einen schweren Stand haben und ihre Umsetzung eine eher unwahrscheinliche Leistung ist*. Im Folgebeitrag loten wir Möglichkeiten aus, wie die Wahrscheinlichkeit der integrierten Versorgung allen Unwahrscheinlichkeiten zum Trotz erhöht werden könnte.

\section{Erreichbarkeitsprobleme}

Obwohl heute kaum jemand die Attraktivität und Notwendigkeit integrierter Versorgungsmodelle bestreitet, ist es zunächst unwahrscheinlich, dass das Thema die einzelnen Organisationen des Gesundheitswesens erreicht, d.h. bei diesen auf Anhieb ein hohes Mass an Aufmerksamkeit findet. Diese Unwahrscheinlichkeit ist auf die spezifische Form der Wertschöpfung im Gesundheitswesen zurückzuführen. Diese ist durch grosse Ungewissheit gekennzeichnet und wird zu einem wesentlichen Teil in der unmittelbaren Interaktion zwischen Medizin, Pflege und Patient erbracht. Dieser Fokus auf die unmittelbare Arzt-Pflege-Patienten-Interaktion dient der Stabilisierung eines fragilen, unsicheren Behandlungsprozesses, hat aber zur Folge, dass für die Klärung grundlegender organisatorischer Fragen zur Arbeitsteilung zwischen Bereichen, Kliniken oder gar anderen Organisationen im Alltag oft zu wenig Platz bleibt [6]. Der Aufbau integrierter Versorgungsmodelle setzt folglich voraus, dass es den Leistungserbringern gelingt, sich vom Arbeitsalltag ein Stück weit $z u$ distanzieren, um die Erfolgsvoraussetzungen des eigenen Wertschöpfungsprozesses und insbesondere seine Einbettung in eine übergeordnete Versorgungslandschaft zu thematisieren. Diese Reflexion des eigenen Wertschöpfungsprozesses und seiner künftigen Ausrichtung ist allerdings ein Kraftakt, der erheblich Zeit und Energie in Anspruch nimmt und stets in Konkurrenz mit dem Sog des operativen Alltags steht. Insbesondere bedingt sie neue, darauf zugeschnittene Kommunikationsprozesse, in denen die Frage nach Sinn, Zweck, künftigen Erfolgsvoraussetzungen und Einbettung des eigenen Wertschöpfungsprozesses bereichsübergreifend adressiert werden kann. Fehlen einer Gesundheitsorganisation die kommunikativen Voraussetzungen für derartige strategische Überlegungen, ist es unwahrscheinlich, dass sie das Thema einer organisationsübergreifenden $\mathrm{Zu}$ sammenarbeit intensiv genug bearbeiten kann.

\section{Verständigungsprobleme}

Selbst wenn es Gesundheitsorganisationen gelingt, die Möglichkeiten und Grenzen ihrer eigenen Wert- schöpfung aus einer gewissen Distanz zum operativen Alltag zu reflektieren und sich für organisationsübergreifende Versorgungsformen zu öffnen, ist es unwahrscheinlich, dass sich die am Netzwerk beteiligten Professionsgemeinschaften verstehen. Typische Professionsgemeinschaften im Gesundheitswesen sind Mediziner, Pflegefachpersonen oder Angestellte der Verwaltung. Eine Professionsgemeinschaft zeichnet sich dadurch aus, dass ihre Mitglieder im Laufe ihrer Ausbildung, praktischen Tätigkeit und fortlaufenden Interaktion (z.B. auf Fachtagungen) eine kollektive Praxis in Form geteilter Kompetenzen, Wissensbestände, Sprachregelungen, Identitäten und Erfolgsvorstellungen entwickeln. In der Durchführung ihrer Praxis bezieht sich eine Professionsgemeinschaft zum Beispiel auf gemeinsame Werkzeuge, Dokumente, Sprachregelungen und Problemlösungsmuster [7]. Aus dem Blickwinkel der integrierten Versorgung geht es nun darum, die hochspezialisierte Praxis der verschiedenen Professionsgemeinschaften organisationsübergreifend fallspezifisch jeweils neu zu integrieren, um den betroffenen Patienten interdisziplinär zu behandeln und innovative, wirksame und möglichst effiziente Patientenpfade zu entwickeln. Innovation und Patientennutzen in der integrierten Versorgung entsteht in einem fortlaufenden interdisziplinären Kommunikationsprozess an den Schnittstellen der Praxis der beteiligten Professionsgemeinschaften (siehe auch [8]). Kommunikative Verständigung innerhalb einer Professionsgemeinschaft gelingt jedoch eher als Verständigung zwischen Professionsgemeinschaften [7]. Während Kommunikation innerhalb einer Professionsgemeinschaft auf eine geteilte Praxis zurückgreifen kann, entstehen zwischen den Professionsgemeinschaften Verständigungslücken, die unter anderem auf divergente Wissensbestände, Sprachregelungen, Entscheidungsmuster und Erfolgsvorstellungen zurückzuführen sind. In der Praxis äussern sich diese Verständigungslücken in sogenannten «Rivalitäten», «Abgrenzungsmentalitäten» oder im «Gärtchendenken» [6] zwischen den Berufsgruppen. Eine Re-Integration der kollektiven Praxis über Professions- und Organisationsgrenzen hinweg wird damit zu einer höchst anspruchsvollen Leistung, die nur selten beiläufig, d.h. ohne zusätzliche, sorgfältig orchestrierte kommunikative Arrangements gelingt.

\section{Verbindlichkeitsprobleme}

Selbst wenn sich die Professionsgemeinschaften verstehen, spricht einiges dafür, dass sie sich schwertun, sich auf eine dauerhafte Kooperation mit aller Verbindlichkeit einzulassen. Zunächst ist eine Zusammenarbeit im Netzwerk nämlich nur um den Preis eines teilweisen Verlusts von Handlungsautonomie zu haben, was bei vielen Professionen auf wenig Begeisterung stossen könnte. Eine patientenzentrierte Kooperation bedingt nämlich, dass die Leistungserbringer ihre Praktiken aufeinander abstimmen, zum Beispiel in sachlicher (z. B. Einführung gemeinsamer kommunikationstheoretische Überlegungen [5]. 
Behandlungsstandards, Werkzeuge und IT-Systeme), sozialer (z. B. Einschränkung der für eine Behandlung in Frage kommender Leistungsanbieter), zeitlicher (z.B. rechtzeitige Übermittlung von Patientendaten) und räumlicher (z.B. Verlagerung der Wertschöpfung in geteilte Behandlungszentren) Hinsicht. Ähnliche Einbussen von Handlungsfreiheit treffen die Patienten, welche die einzelnen Leistungserbringer nur noch bedingt frei wählen können, sobald sie sich den Spielregeln eines Netzwerks unterwerfen. Im Umgang mit diesen Einbussen wahrgenommener Wahlfreiheit liegt eine der zentralen Herausforderungen des Managements integrierter Versorgungsnetzwerke. Die Gratwanderung liegt darin, die Eigenständigkeit der beteiligten Akteure zu respektieren und gleichzeitig verbindliche Spielregeln für eine Kooperation in einem übergeordneten Ganzen zu schaffen [9]. Es wird kaum überraschen, dass dieser Spagat äusserst voraussetzungsreich ist.

Darüber hinaus ist es wahrscheinlich, dass das verbindliche Engagement für ein integriertes Versorgungsnetzwerk an seiner sachlichen und sozialen Komplexität scheitert. Mit sachlicher Komplexität ist gemeint, dass im Netzwerk mehr und variantenreichere Expertise für die Behandlung eines spezifischen Krankheitsverlaufes mobilisiert werden kann. Im Netzwerk schaut eine grössere Anzahl von Professionsgemeinschaften aus unterschiedlichen Disziplinen auf einen Patienten als in einer fragmentierten Wertschöpfung. Diese Diversität ermöglicht es dem Netzwerk, variantenreicher zu diagnostizieren, und mehr therapeutische Optionen zu entwickeln. Diversität ist der Komplexität heutiger Krankheitsbilder, z. B. chronischer Erkrankungen, durchaus angemessen: Integrierte Versorgungsnetze antworten auf die Komplexität dieser Erkrankungen, indem sie die Komplexität der Behandlung und damit ihre Eigenkomplexität erhöhen. Das grosse Potential integrierter Versorgungsnetze liegt demnach in der Befolgung des Rezepts: «It takes variety to control variety» [10]. Die Anwendung dieses Rezepts wirft allerdings die Frage auf, wie man mit der erhöhten Systemvarietät angemessen umgehen kann. Wie einigt man sich im Netzwerk, wenn aufgrund der unterschiedlichen Praxis der beteiligten Professionsgemeinschaften mehrdeutige, vielleicht sogar widersprüchliche Diagnosen und Therapievarianten generiert werden? In welchen Situationen lässt man Varietät $\mathrm{zu}$, und in welchen nicht? Wie kommt man in diesem Kontext zu gemeinsamen, von allen getragenen Entscheidungen?

Auf die sachliche Komplexitätssteigerung folgt unweigerlich eine soziale: Je mehr Professionen aus unterschiedlichen Organisationen zusammenwirken, desto wahrscheinlicher wird es, dass sich der eine oder andere anders verhält als vom Netzwerk erwartet: gemeinsame Behandlungsleitlinien werden nicht respektiert, Diagnosen werden nicht sauber dokumentiert, die gegenseitige Transparenz, die eine Zusammenarbeit im Netzwerk zwangsläufig mit sich bringt, wird von einzelnen Akteuren opportunistisch ausgenutzt. Opportunistisches Verhalten kann in der integrierten Versorgung nicht ausgeschlossen werden, da die beteiligten Akteure oft Partner und Konkurrenten zugleich sind. Während man im einen Behandlungsfeld kooperiert, wetteifert man im anderen. Für alle Beteiligten (inklusive Patienten) steigt damit die Unsicherheit über die Verlässlichkeit und Vertrauenswürdigkeit ihrer Partner. Gelingt es dem Netzwerk nicht, verbindliche Spielregeln für den Umgang mit diesen Spannungsfeldern zu entwickeln, ist es wahrscheinlich, dass die Beteiligten das Interesse an einer Zusammenarbeit trotz besten $\mathrm{Ab}$ sichten schnell wieder verlieren.

\section{Fazit und Ausblick}

Das schweizerische Gesundheitswesen ist mit einer Reihe von gesellschaftlichen und versorgungspolitisch relevanten Veränderungen konfrontiert. Neue integrierte Versorgungsmodelle gelten weit um als eine Möglichkeit, diesen Herausforderungen vorausschauend zu begegnen. In diesem Beitrag argumentieren wir, dass solche Modelle trotz aller zustimmenden Rhetorik in ihrer Umsetzungspraxis eine höchst unwahrscheinliche Leistung darstellen:

- Zunächst ist es unwahrscheinlich, dass das Thema einer organisationsübergreifenden $\mathrm{Zu}$ sammenarbeit die Leistungserbringer im Gesundheitswesen erreicht. Die anspruchsvolle Arbeit am Patienten absorbiert in der Regel so viel Energie, dass Fragen einer bereichs- oder gar organisationsübergreifenden Zusammenarbeit im operativen Alltag oft zu kurz kommen.

- Selbst wenn es den Professionsgemeinschaften gelingt, ihre Aufmerksamkeit in die Richtung einer organisationübergreifenden Zusammenarbeit zu öffnen, ist es unwahrscheinlich, dass sie sich verstehen. Im Zuge ihrer Ausdifferenzierung haben sie hochspezifische Praktiken entwickelt, die für die Netzwerkpartner nur mit grossem Kommunikationsaufwand nachvollziehbar sind. - Schliesslich ist es unwahrscheinlich, dass sich die beteiligten Akteure (inklusive Patienten) auf eine Zusammenarbeit im Netzwerk mit aller Verbindlichkeit einlassen. Netzwerke schränken (wahrgenommene) Wahlfreiheiten ein und erzeugen eine sachliche und soziale Eigenkomplexität, die für die Kooperationspartner nur schwer zu bewältigen ist.

Trotz dieser Unwahrscheinlichkeiten besteht wenig Zweifel an der Notwendigkeit integrierter Versorgungsmodelle. Umso wichtiger wird es, über die grundlegenden Voraussetzungen ihres Gelingens nachzudenken. Im kommenden Beitrag loten wir daher Möglichkeiten aus, wie die Wahrscheinlichkeit gelingender organisationsübergreifender Kooperation im Gesundheitswesen allen Unwahrscheinlichkeiten zum Trotz gesteigert werden könnte. 


\section{Literatur}

1 Sottas B, Brügger S. Ansprechstrukturen: Perspektivenwechsel und Grenzverschiebungen in der Grundversorgung. Zürich: Careum; 2012.

2 Schweizerische Gesundheitsdirektorenkonferenz und Bundesamt für Gesundheit. Neue Versorgungsmodelle für die medizinische Grundversorgung. Bern: Bericht der Arbeitsgruppe «Neue Versorgungsmodelle für die medizinische Grundversorgung» von GDK und BAG; 2012. S. 21.

3 Schweizerische Akademie der Medizinischen Wissenschaften. Projekt «Zukunft Medizin Schweiz» Phase III. Die zukünftigen Berufsbilder von ÄrztInnen und Pflegenden in der ambulanten und klinischen Praxis. Bericht aus dem Jahr 2007 und Kommentar 2011. Basel: SAMW; 2011. S. 27.

4 Rüegg-Stürm J, Tuckermann H, Bucher S, Merz J, Von Arx W. Management komplexer Wertschöpfungsprozesse im Gesundheitswesen: Vernetzung beginnt in der Organisation. In: Amelung V, Sydow J (Hrsg.).

Vernetzung im Gesundheitswesen: Wettbewerb und Kooperation. Stuttgart: Kohlhammer; 2009.

S. 181-210.
5 Luhmann N. Die Unwahrscheinlichkeit der Kommunikation. In: Luhmann N (Hrsg.). Soziologische Aufklärung 3. Opladen: Westdeutscher Verlag; 1981. S. 25-34.

6 Tuckermann H, Rüegg-Stürm J. Sind Spitäler wandelresistent? Schweiz Ärztezeitung. 2007;88:1895-9.

7 Brown JS, Duguid P. Knowledge and organization: A social-practice perspective. Organization Science. 2001;12(2):198-213.

8 Gibbons M, Limoges C, Nowotny H, Schwartzman S, Scott $\mathrm{P}$, Trow M. The new production of knowledge. London: Sage; 1994.

9 Wimmer R. Von der Hierarchie zum internen Netzwerk. Hernsteiner. 1997;2:14-20.

10 Ashby WR. Requisite variety and its implications for the control of complex systems. Cybernetica. 1958;1(2):83-99. 